\title{
Experimental Research on the Effect of Gasoline Quality on Vehicle Emission and OBD System
}

\author{
WU $\mathrm{Di}^{1, \mathrm{a}}$ \\ ${ }^{1}$ China Automotive Technology\&Research Center Automotive Testing and Research Institute, \\ Tianjin 300300, China \\ awudi@catarc.ac.cn
}

Keywords: Vehicle emission; Gasoline quality; on-Board Diagnostics (OBD); Sulfur content; Olefins Content; Exhaust Emissions; Catalytic Converter

\begin{abstract}
With the surging emissions standards for cars and strict and the application of advanced emission control technology, the influence of fuel quality on vehicle emissions is increasingly important. As a result, automobile, petrochemical industry in the United States, Japan, Europe and other countries and regions have cooperated on the research on the car, engine and fuel system. Key technical indicators of fuel limit corresponding to gradual enhancement of the vehicle emission regulations are put forward. With the issue of country's III and IV phase vehicle emission standards, fuel specifications that meet the discharge standard should be established.
\end{abstract}

\section{Effect of gas composition and properties on vehicle performance and emissions}

Gasoline is mainly composed of three types of hydrocarbons, namely olefin, alkanes and aromatic hydrocarbons. Because gasoline itself contains a certain amount of sulfur in crude oil, sulfur generally can't be completely removed during the gasoline refining, so it will contain a certain amount of sulfur in gasoline.

\section{Effect of the sulfur content in gasoline on vehicle emissions}

The sulfur in the gasoline can obviously reduce the efficiency of the catalysts in the catalytic converters. Sulfur can also adverse effects on the high temperature exhaust gas oxygen sensor. [1] To reduce sulfur content in fuel oil can directly reduce the emissions of vehicles with catalytic conversion device significantly. Impact of the sulfur on the vehicle emissions of pollutants has been researched with extensive experiments.

\section{Effect of the benzene content in gasoline on vehicle emissions}

Benzene naturally occur in the crude oil composition, it is also catalytic reforming products, a high octane number gasoline components. It is also known a carcinogen.

Benzene content controlling of gasoline is the most direct limit from the evaporate emission and effective method to vent of benzene emissions. Studies have shown that the benzene content in gasoline with exhaust gas of benzene emissions is produced by linear relationship, benzene emissions also have very high contribution to gasoline aromatic compounds. Many countries law makers have realized controlling benzene content in gasoline is an effective method to reduce benzene harm to mankind.

\section{Effects of gasoline volatile on vehicle}

Reasonable volatile is critical for gasoline engine work in both performance and emissions. Gasoline volatile can be determined by vapor pressure and distillation range.

Gasoline vapor pressure should be controlled according to the season, make different temperatures have different volatile. At high temperature, it is necessary to control the vapor pressure strictly to avoid problems at high temperature, such as gas resistance or canister overload. Control of high temperature vapor pressure is also important to reduce evaporate emission. To warm machine starting smoothly and get good machine performance, a high vapor pressure is needed at low temperature. Proper reduce of the vapor pressure of gasoline can reduce the HC emissions and CO emissions of vehicles. 


\section{Simulation of multi-component evaporation model based on the gasoline engine working process}

This chapter mainly introduces the three components of oil droplets evaporation model gasoline and oil film evaporation model of development. [2]Determine the proportion of alternative model by gasoline fuel test components. Use the test results to verify the correctness of the model. The model developed is embedded in CFD (computational fluid dynamics) program, to simulate and analyze gasoline engine cold start conditions volatile effects on mixture formation and combustion process.

Mathematical model of three-dimensional CFD simulation in gasoline engine working process

Combustion system and geometric shape is complex, the internal flow field is in rapidly changing, working medium has a strong compatibility, burning with high temperature and high pressure, the fuel oil mixture also involve two phase flow. In recent years, with the rapid development in the d high-speed and large-capacity computer technology, as well as the progress of computational fluid dynamics and combustion theory, CFD has become the important means in the process of engine research and development. Through three-dimensional CFD simulation can get the engine working process flow, the detailed information of the mixture formation and combustion for understanding the experiment phenomenon, provides theory basis for optimization of engine design parameters.

(1) continuity equation

$$
\frac{\partial \rho_{g}}{\partial t}+\frac{\partial}{\partial x_{j}}\left(\rho u_{j}\right)-S_{m}=0
$$

(2) momentum conservation equation

$$
\frac{\partial}{\partial t}\left(\rho_{g} u_{t}\right)+\frac{\partial}{\partial x_{i}}\left(\rho_{g} u_{i} u_{j}-\tau_{i j}\right)+\frac{\partial p}{\partial x_{i}}-\rho_{g} g \frac{x_{i}}{|x|}-F_{t}^{s}=0
$$

In the type, $x_{i}$ represents the direction of three coordinates $x_{1}, x_{2}, x_{3}$ in the three-dimensional rectangular coordinate system, $g$ is the gas pressure, $F^{*}$ is acceleration of gravity, $\tau_{i j}$ for the viscous stress tensor of droplets of the fluid micro fluid control surface, of Newtonian fluid:

$$
\tau_{i j}=2 \mu_{g}\left(S_{i j}-\frac{1}{3}\left(\sum \frac{\partial u_{k}}{\partial x_{k}}\right) \delta_{i j}\right)
$$

(3) energy conservation equation

$$
\frac{\partial}{\partial t}\left(\rho_{g} h\right)+\frac{\partial}{\partial x_{j}}\left(\rho_{g} u_{j} h\right)-\frac{\partial}{\partial x_{j}}\left(\frac{\lambda_{g}}{c_{p g}} \cdot \frac{\partial h}{\partial x_{j}}\right)-\frac{\partial}{\partial x_{j}}\left(\tau_{i j} u_{i}\right)-\frac{\partial p}{\partial t}-S_{h}=0
$$

In the type, $S_{h}$ said source term of energy, such as the droplet evaporation heat flux, the $c_{p g}$ for gas specific heat at constant pressure, $\lambda_{g}$ for gas coefficient of thermal conductivity, $h$ is the stagnation enthrall, the definition expression is:

$$
h=c_{p g} T+\frac{u_{i}^{2}}{2}
$$

\section{Multi-component evaporation model}

Fuel evaporation process is an important part of engine mixture form, which directly affects the combustion and emission characteristics. [3]Especially in the cold start process, the effect is more obvious, fuel evaporation too slow may lead to starting abnormal or even to fail. Most of the fuel evaporation model consider fuel for single component, but the actual fuel such as gasoline or diesel have hundreds of components, which is a mixture of various volatile components. If the environment temperature is very high, droplet evaporation speed is fast, fuel composition is not obvious when the evaporation process of the differences between using then single-component model was acceptable. But if the environment temperature is lower, slower droplet evaporation, the 
clear difference between components, such as use single-component model will not be able to accurately simulate the actual gasoline evaporation process in cold start process, multi-component evaporation model must be used for different components of the evaporation process.

\section{Multi-component droplet evaporation model}

Based on the classic film theory, this model simulate the oil droplets in relative gas movement in the process of heat and mass transfer. Film theory assumes that the oil surface and the surrounding gas is focused on the heat and mass transfer resistance of virtual gas film with a certain thickness, the thickness of the film should guarantee that thermal conductivity formed by the molecular motion and diffusion are in the same rate with the actual medium oil droplet surface quality and heat transfer and mass transfer between the airflow.

Model description is as follows:

1) assume that the gas film for steady state, and the oil droplets is always spherical;

2) components inside the droplet is uniformly distributed, and evaporation process are independent of each other;

3) consider the interactions among components in oil droplets;

4) consider forced convection movement and Stefan flow phenomenon in the process of oil droplets evaporation.

(1) the multi-component droplet evaporation of multi-component mass transfer oil droplets evaporation rate is:

$$
m_{d}=\sum_{k=1}^{K} m_{k}
$$

In the type, $m_{d}$ is for droplet total rate of evaporation, $m_{k}$ for evaporation rate in the oil droplets components:

$$
\dot{m}_{k}=2 \pi r_{d}(\rho D)_{g k} S h_{k}^{*} \ln \left(1+B_{Y K}\right)
$$

According to the theory of membrane and the above assumptions, the virtual any component in the gas film $\mathrm{k}$ in mass flow rate on the surface of the free kick is:

$$
J_{k}=4 \pi r^{2}\left[-\rho_{g} D_{g} \frac{d Y_{k}}{d r}+\rho_{g} v_{r} Y_{k}\right]
$$

(2) Multi-component droplet evaporation heat transfer

The overall heat transfer rate of oil droplets from the outside world

$$
\dot{Q}_{d}=m_{d}\left(\frac{\overline{c_{p d}}\left(T_{\infty}-T_{d}\right)}{\exp \left(\frac{m_{d} \overline{c_{p d}}}{2 \pi r_{d} \lambda_{g} N u^{*}}\right)-1}-L\left(T_{d}\right)\right)
$$

\section{Multi-component oil film evaporation model}

Assumes that the component ratio remain initial state of fuel oil film, and ignore the interaction between components, only solve the mass conservation equation of the oil film as a whole to avoid solving the continuity equation of each component in the fuel oil, which can simplify the model, and reduce the amount of calculation, but the model precision is reduced.

The total oil film evaporation rate is:

$$
\dot{m}_{f}=\sum_{k=1}^{K} \dot{m}_{f, k}
$$

The quality of each constituent evaporation rate is:

$$
\dot{m}_{f, k}=\max \left(\dot{m}_{f, k 1}, \dot{m}_{f, k 2}\right)
$$


In the type, calculating formula for $m_{f, k}$ is:

$$
\dot{m}_{f, k 1}=-\rho_{v, k} u_{g / /} S t_{m k}\left(\frac{Y_{k}-Y_{s, k}}{1-Y_{s, k}}\right)
$$

\section{Effect of fuel quality on the OBD system}

\section{OBD system introduction}

OBD is the abbreviation of On-Board Diagnostics, namely "on-board self-diagnosis system”. It mainly monitors component or system related to emissions. If found the component or system emissions caused by the degradation or failure exceed bid, OBD system will light MIL, and control module (PCM) will to store fault information in memory, which can be read from PCM through certain procedures. According to the fault code, maintenance personnel can quickly and accurately determine the nature and the location of a fault[4].

OBD system can ensure the service life of the car in the whole discharge no more than in the OBD regulatory requirements, dramatically reduce emission due to the failure of in-use vehicle standard. At the same time, the use of OBD system monitoring information in the I/M check, the car inspection and maintenance procedures can be simplified, thus reduce the time interval between the failure and maintenance. In addition, the implementation of the OBD can ensure the vehicle pollution control device production consistency, reduce the scatter difference between components and systems, and improve its durability, guarantee the in-use vehicle emission compliance within the service life.

\section{Test results and analysis}

As for the vehicles involved in the project, eight cars made some OBD function verification test of the project, among which five completed all the validation project.

Because this project is still in research stage, understanding of each unit of OBD validation process is not very clear, the validation work was done with the help of relevant foreign partners, and the corresponding simulation equipment need to carry from abroad, preparation work is very difficult, OBD validation project of the eight demo car is not very complete. With the test times increases, the project team personnel understanding of OBD regulations better and better, which has laid a solid foundation for Beijing in advance of OBD project .

Related content in Euro III and euro IV regulations for OBD project is not much, which would increase difficulty in emissions standards in the process of the implementation of the corresponding part. OBD legislation is important constituent the implementation of the developed countries such as Europe and the United States, which is also one of the effective means of in-use vehicle management in the process of consistency check and government departments. But Europe and the United States and other countries have a sound in-use vehicle management mode, including the annual inspection, road inspection, user management and defect recall system, etc., while although China promulgated the measures for the recall of defective vehicles but because it just begin, still need a long time to complete.

\section{Summary}

The quality of vehicle fuel and emission control technology has been closely fused, which influence each other. Continuously-tighten emissions requirements need a dramatic rise in fuel quality. When the fuel components and technical indicators have gotten obvious change, it will accelerate the progress of auto technology, and promote the wide application of high and new technology, thus to meet stringent emission requirements better. As car exhaust emission standards becomes more and more stringent, gasoline oil increasingly occupies increasing important impact on vehicle emissions. 


\section{References}

[1] Rodica Faucon. European standards and regulations and car manufacturers' needs for quality fuels. SAE papers. 2004

[2] J.Turex Timothy. Interaction of Sulfur with Automotive Catalysts and the Impact on Vehicle Emissions-A Review SAE technical Paper series 1999

[3] Fukui H, Komoriya H, Shimizu T. Effects of octane number on stratified charge combustion in a direct- injection gasoline engine. SAE Paper 2001-01-1964

[4] Saitoh K, Hamasaki M. Effects of sulfur, aromatics, T50, T90and MTBE on mass exhaust emission from vehicles with advanced technology-JCAP gasoline step II report. SAE Paper 2003-01-1905 